\title{
Numerical analysis of non-isothermal infiltration process for fabricating magnesium matrix composite
}

\author{
Su Lizheng ${ }^{1, \text { a }}$, Bai Yunfei ${ }^{1, b}$, Li Bo ${ }^{1, c}$, Zhou Yapeng ${ }^{1}$ and Wang Kejun ${ }^{1}$ \\ ${ }^{1}$ Xi'an Electronics Engineering Research Institute, Xi'an, 710100, P.R.China

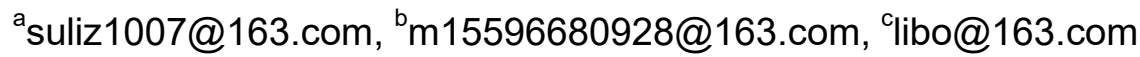

\begin{abstract}
Keywords: Magnesium matrix composite; Non-isothermal infiltration; Porous perform; Numerical analysis.
\end{abstract}

Abstract. For the control and adjustment of the infiltration quality of the magnesium alloy liquid in porous perform, the coupling effect of temperature and pressure should be considered simultaneously. The thermodynamic characteristics of the local region can be adjusted by changing the boundary temperature of the porous performs. In this study, the mathematical model of the flow and heat transfer process of magnesium alloy liquid in carbon fiber perform was established. The numerical solution of the partial differential equation system is developed. The effects of the constant heating temperature and the linear heating temperature on the infiltration characteristics of the porous perform are investigated. The results showed that the linear non-isothermal heating condition could change the flow characteristics of the magnesium alloy liquid close to the inner wall of the porous perform. The fluid velocity along the flow direction tended to be average in the region close to the heating wall. Imposing temperature gradient on porous media was an effective way to regulate and control the infiltration quality. The numerical predictions were compared with the experiment data, and good agreement had been found between them. In addition, the numerical models could be developed to predict the appearance of defects in the end product and to study the evolution of the deformation of fibrous preform during metal infiltration.

\section{Introduction}

Magnesium matrix composites have excellent performance as lightweight and high-performance structural materials, which can be used in airborne radar structural parts [1,2]. The extrusion directly following vacuum infiltration technique (EFVI) is one of the most important processes for preparing magnesium matrix composites. In EFVI, melt infiltration is a key process whereby molten magnesium alloy can infiltrate completely into the porous perform [3,4]. When the liquid magnesium alloy flows through the preform, its thermodynamic properties also change with the alloy liquid flow and temperature conditions. But, due to the difficulties presented by the high temperature and pressure employed during the liquid infiltration processes, it is difficult to visualize the melt flow behavior and the coupling phenomena in the fiber perform by conventional techniques. Numerical tools such as finite element and finite difference methods are applied. Ballmes H.K et al [5] investigated preform infiltration based on the thermal conductivity and Darcy's law by finite element method (FEM). Qi L.H et al [6] simulated the temperature fields and the infiltration fields by the finite element method and the finite difference method, respectively. In these studies, the research on the infiltration characteristics of liquid metal in porous media is mainly focused on the isothermal system. However, little attention has been paid to the study of fluid flow and thermodynamic properties of porous perform under non-isothermal temperature field. During infiltration, the fluid is strongly dependent on the temperature and pressure of the porous preform. The uniformity of the temperature field will directly affect the fluid properties Therefore, it is necessary to consider the coupling effect of temperature gradient and fluid flow on the properties of the liquid magnesium alloy. In this study, the mathematical model of coupling the flow and heat transfer of the liquid magnesium alloy in the 
porous perform is established. The properties of liquid magnesium alloy flow in porous perform under non-isothermal temperature field were numerically analyzed.

\section{Model establishment}

\section{Physical model}

The porous cylindrical model with high $\mathrm{H}$ and radius $\mathrm{R}$ is established. The liquid magnesium alloy flows from the top of the cylinder and flows out from the bottom. The two heating modes are considered. The model one is shown in Figure 1(a). The left side of the isotropic and homogeneous porous preform maintains the constant temperature $\mathrm{T}_{\mathrm{C}}$ and the right side temperature is the constant temperature $\mathrm{T}_{\mathrm{H}}$. The interior of the preform is linearly increased from left to right. The model is consistent with the fact that the magnesium alloy and preform are separately heated separately. The hear temperature of the preform during the heating process is higher than that of the edge. The other model is shown in Figure 1(b). The temperature of the lower and right sides of the preform is kept constant from $T_{C}$ to $T_{H}$. The temperature increases linearly from $T_{C}$ to $T_{H}$ and increases linearly from $\mathrm{T}_{\mathrm{C}}$ to $\mathrm{T}_{\mathrm{H}}$ from left to right. The model is consistent with the magnesium alloy and preform is heated at the same time in the device. In order to study the coupling behavior of liquid magnesium alloy in the fiber preform. The mathematical model of the physical model is composed of the continuity equation, the momentum equation, the energy equation and the state equation.

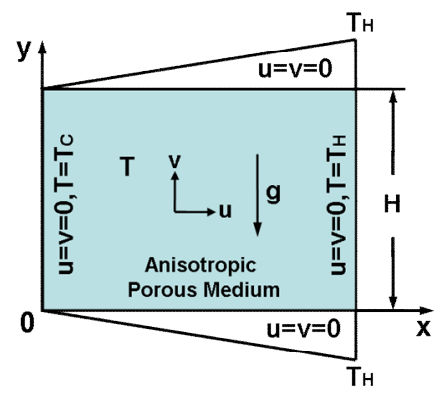

(a) model one

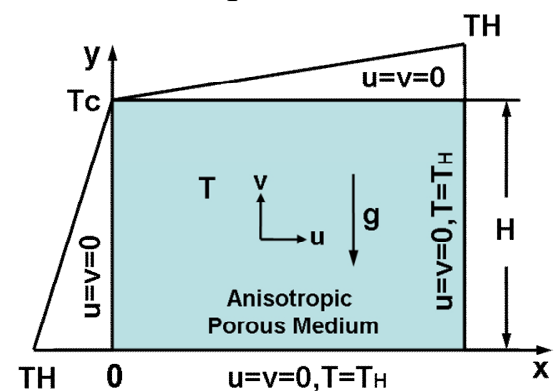

(b) model two

Fig.1 Physical model

\section{Mathematical model}

\section{The continuity equation}

Considering the fluid body with volume $\tau$ and surface area $\mathrm{A}$ in the porous media, and utilizing the mass conservation principle of the fluid, then

$$
\frac{\partial}{\partial t} \iiint_{t} \rho \varepsilon d \tau+\oiint n \rho V \varepsilon d A=0
$$

Where, $t$ is the infiltration time, $n$ is the unit normal vector of the elemental area $d A$ on the fluid body surface, $\rho$ is the metal density, $\varepsilon$ is the volume fraction occupied by the fluids.

According to $\partial \varepsilon / \partial t=0$ and the particle differential equation, the continuity equation Eq. 1 by using Gauss law has the following form:

$$
\varepsilon \frac{\partial \rho}{\partial t}+\rho \nabla(\varepsilon V)=0
$$

\section{The motion equation}

In a porous medium, the flow of fluid satisfies Darcy's law:

$$
u=-\frac{K}{\mu} \nabla P
$$


Where, $P$ is the fluid pressure, $\mathrm{Pa}, K$ is the permeability coefficient, $\mu$ is the fluid dynamic viscosity. The permeation coefficient of the preform is determined by the formula in Eq. 1.

$$
K=\frac{d_{f}^{2}(1-\phi)^{3}}{125 \phi}
$$

Where, $\phi$ is the volume fraction of preform, $d_{f}$ is the fiber diameter, $\mu \mathrm{m}$.

\section{The infiltration heat transfer equation}

It is assumed that the porosity of the preform is isotropic, regardless of radiative heat transfer and viscous dissipation, and assumes local thermal equilibrium, i.e., where the sums represent the temperatures of the solid and fluid phases, respectively. Respectively, for solid and fluid phase energy balance calculation, as follows:

For the solid phase, the energy balance is calculated as follows:

$$
(1-\phi)(\rho C)_{s} \frac{\partial T}{\partial t}-(1-\phi) \nabla\left(\lambda_{s} \nabla T\right)=0
$$

For the fluid phase, the energy balance is calculated as follows:

$$
\phi\left(\rho C_{p}\right)_{f} \frac{\partial T}{\partial t}+\left(\rho C_{p}\right)_{f} u \nabla T-\phi \nabla\left(\lambda_{f} \nabla T\right)=0
$$

Where, $C$ is the specific heat of the solid, $J /(\mathrm{kg} \cdot \mathrm{K}), C_{p}$ is the specific heat of the constant pressure of the fluid, $J /(\mathrm{kg} \cdot K), \lambda$ is the thermal conductivity, $W /(m \cdot K) . C$ and $C_{p}$ is a function of temperature, $\lambda$ is the function of temperature and pressure.

\section{The simplification of mathematical model}

The momentum Eq. 4 is directly substituted into the continuity Eq. 3 and energy balance Eq. 5 and Eq. 6, the above equations can be simplified as follows:

$$
\begin{gathered}
\frac{\partial\left(\partial \rho_{f} \phi\right)}{\partial t}+\nabla\left(\rho_{f} \frac{\kappa}{\mu} \nabla P\right)=0 \\
(1-\phi)(\rho C)_{s} \frac{\partial T}{\partial t}-(1-\phi) \nabla\left(\lambda_{s} \nabla T\right)=0 \\
\phi\left(\rho C_{p}\right)_{f} \frac{\partial T}{\partial t}+\left(\rho C_{p}\right)_{f} u \nabla T-\phi \nabla\left(\lambda_{f} \nabla T\right)=0
\end{gathered}
$$

For the apparent thermal conductivity of the preform filled with magnesium alloy, the following formula can be used:

$$
\lambda_{m}=\lambda_{s}^{1-\phi} \lambda_{f}^{\phi}
$$

Where, $\lambda_{f}$ is the magnesium alloy liquid thermal conductivity, $W /(m \cdot K), \lambda_{s}$ is the prefabricated solid skeleton thermal conductivity, $W /(m \cdot K)$.

\section{The boundary conditions}

The given pressure of fluid inlet and outlet belong to the first boundary condition. The pressure gradient at the other boundary is 0 , that is $\nabla P=0$. It belongs to the second kind of boundary condition.

For the boundary conditions of heat transfer, the temperature of the fluid is given at the fluid inlet. The heat transfer at the fluid outlet is fully developed, i.e. $\nabla T=0$. It belongs to the second boundary condition. While, the temperature distribution function is given at the cylinder boundary. 


\section{The simulation conditions and programs}

In this paper, the flow and heat transfer characteristics of the fluid in the porous preform are studied by taking the flow process of the liquid magnesium alloy in the carbon fiber preform as an example. In the calculation, the properties of the calculation area, fluid and preform are shown in Table 1. The heating boundary conditions used in the calculation are shown in Table 2.

Table 1 Characteristics of AZ91D alloy and carbon fiber porous perform

\begin{tabular}{c|c|c|c|c|c}
\hline \hline $\begin{array}{c}\rho \\
\mathrm{kg} / \mathrm{m}^{3}\end{array}$ & $\begin{array}{c}\mu \\
\mathrm{Pa} \cdot \mathrm{s}\end{array}$ & $\begin{array}{c}\lambda_{f} \\
W /(m \cdot K)\end{array}$ & $\begin{array}{c}\mathrm{C}_{\mathrm{p}} \\
J /(\mathrm{kg} \cdot K)\end{array}$ & $\phi$ & $\begin{array}{c}T_{C} \\
\mathrm{~K}\end{array}$ \\
\hline 1830 & 0.003 & 21 & 1020 & 0.15 & 873 \\
\hline $\begin{array}{c}\mathrm{C}_{\mathrm{f}} \\
J /(\mathrm{kg} \cdot K)\end{array}$ & $\mu \mathrm{m}$ & $W /(m \cdot K)$ & $\mathrm{mm}$ & $\mathrm{H}$ & $P_{c}$ \\
$\mathrm{~mm}$ & $\mathrm{MPa}$ \\
\hline 1720 & 14 & 0.28 & 22.5 & 30 & 0.1 \\
\hline \hline
\end{tabular}

Table 2 Boundary conditions in the simulations

\begin{tabular}{l|l|l}
\hline \hline Boundary & Model one & Model two \\
\hline \multirow{3}{*}{$x=R$} & $\frac{\partial p}{\partial t}=0, \frac{\partial u}{\partial x}=0$ & $\frac{\partial p}{\partial x}=0, \frac{\partial u}{\partial x}=0$ \\
& $T=T_{H}$ & $T=T_{H}$ \\
\hline$y=H$ & $P_{\text {in }}=2.0 P_{C}$ & $P_{\text {in }}=2.0 P_{C}$ \\
& $T=T_{C}+\left(T_{H}-T_{C}\right) * x / R$ & $T=T_{C}+\left(T_{H}-T_{C}\right) * x / R$ \\
\hline \multirow{3}{*}{$=0$} & $\frac{\partial p}{\partial x}=0, \frac{\partial u}{\partial x}=0$ & $\frac{\partial p}{\partial x}=0, \frac{\partial u}{\partial x}=0$ \\
\hline$y=0$ & $T=T_{C}$ & $T=T_{H}-\left(T_{H}-T_{C}\right) * y / H$ \\
\hline \hline
\end{tabular}

For the equations as from (7) to (9) and the boundary conditions as shown in the table, COMSOL Mutiphysic software were used for numerical solution of the two model conditions. In the whole calculation area, the encryption grid is adopted at the inlet boundary $(y=H)$ and the outlet of the fluid. The maximum length of the unit is $10^{-5} \mathrm{~m}$. The convergence condition is relative error for $1 \times 10^{-6}$, and the maximum iteration number is 10 . In this study, only the coupling characteristics of flow and heat transfer under transient conditions are calculated and analyzed.

\section{Results and Discussions}

Figure 2 (a) shows the distribution of temperature field under the condition of model one. There is a temperature gradient in the heating process, and the temperature contour linearly changes from right to left. Fig. 2 (b) shows the temperature field distribution under the condition of model two. The influence of different temperature field conditions on melt flow of magnesium alloy is analyzed as following. 


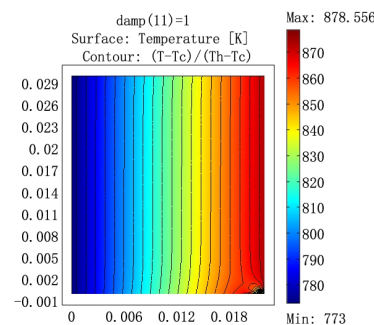

(a) model one

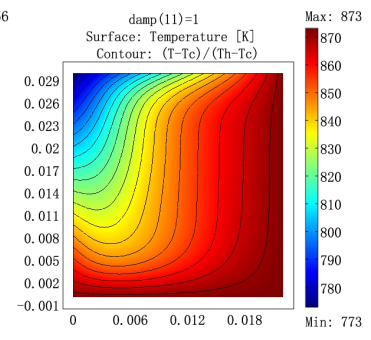

(b) model two

Fig.2 Dimensionless temperature in a porous perform subjected to temperature gradients and subsequent free convection

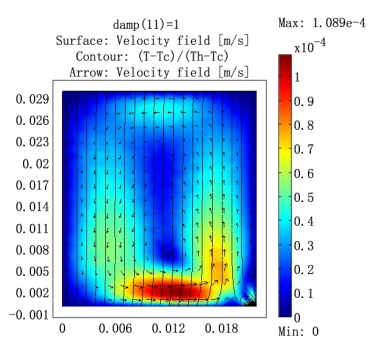

(a)

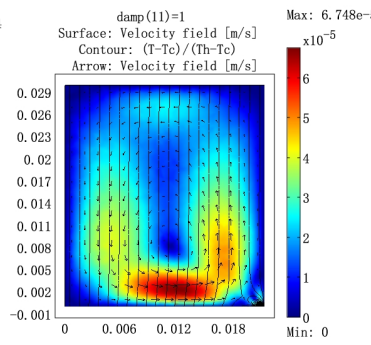

(b)

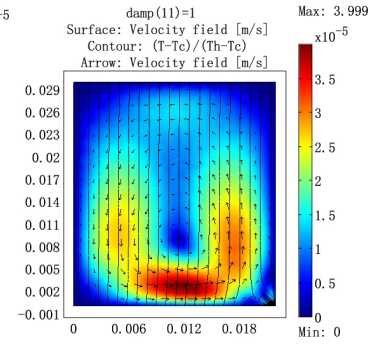

(c)

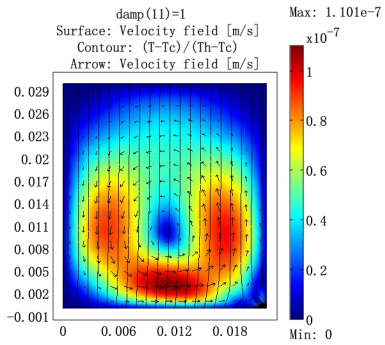

(d)
(a) $\mathrm{T}_{\mathrm{H}}=600^{\circ} \mathrm{C}, \mathrm{Tc}=500^{\circ} \mathrm{C}$
(b) $\mathrm{T}_{\mathrm{H}}=600^{\circ} \mathrm{C}$ $\mathrm{Tc}=600^{\circ} \mathrm{C}$.
(c) $\mathrm{T}_{\mathrm{H}}=600^{\circ} \mathrm{C}, \mathrm{Tc}=575^{\circ} \mathrm{C}$
(d) $\mathrm{T}_{\mathrm{H}}=600^{\circ} \mathrm{C}$,

Fig.3 Velocity field (surface color) and velocity arrows in a porous perform subjected to temperature gradients.

Figure 3 shows the velocity distribution of the temperature gradient applied by the model in the calculation area. It can be seen from the figure that the temperature gradient has a significant effect on the velocity distribution in the calculation region, especially in the temperature influence region. The fluid velocity in the near wall region is faster than that in the central region. The velocity gradient in the flow direction is gradually increased, which is very different from the case where the temperature gradient is not applied. In general, the temperature of magnesium alloy liquid in the region affected by temperature is higher when the preform temperature is higher. As a result, the fluid flow velocity increases with the fluid density and the fluid viscosity decreases. However, the pressure gradient is small in the direction perpendicular to the flow, resulting in a decrease in the velocity of the fluid near the wall. Whereas a peak band of fluid flow velocity occurs at the bottom outlet, as be shown in Figure 3(a). In addition, the flow velocity of the fluid gradually increases with the increase of the temperature difference between $T_{H}$ and $T_{C}$. Without the influence of temperature gradients, the central region of the thermal perturbation of the fluid affected by the boundary expands and the internal flow of the preform gradually becomes uniform, as shown in Fig. 3(d). Comparing Fig.4 with different temperature contours, it can be seen that the flow velocity changes with the change of the temperature field distribution of the preform, and the velocity changes around the percolation outlet are obvious. This part of the region by the external environment temperature has greater impact. By comparing the velocity field distributions at different temperature gradients, it can be seen that the existence of temperature field changes the spatial distribution of the seepage field. When the temperature difference between $\mathrm{T}_{\mathrm{H}}$ and $\mathrm{T}_{\mathrm{C}}$ reaches $100{ }^{\circ} \mathrm{C}$ (see Fig. 4(a)), the maximum flow velocity near the left and bottom reaches $2.747 \mathrm{~mm} / \mathrm{s}$. The flow is counterclockwise circularly, and a large no-flow area appears in the center. As the temperature difference decreases, ie the temperature gradient decreases, the flow velocity decreases. The area of the parcel is gradually reduced. When the $\mathrm{T}_{\mathrm{H}}$ and $\mathrm{T}_{\mathrm{C}}$ are almost equal (see Figure 4(d)), the temperature gradient tends to be uniform and the influence becomes smaller. 


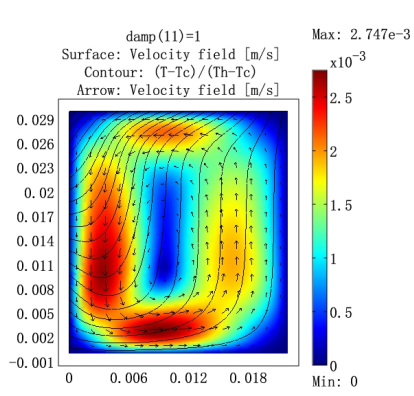

(a)

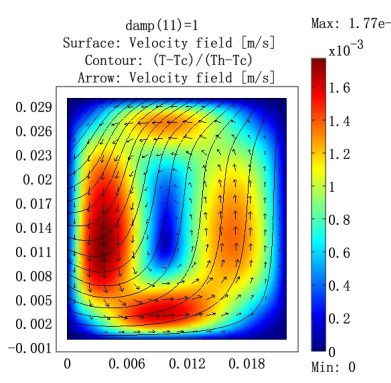

(b)

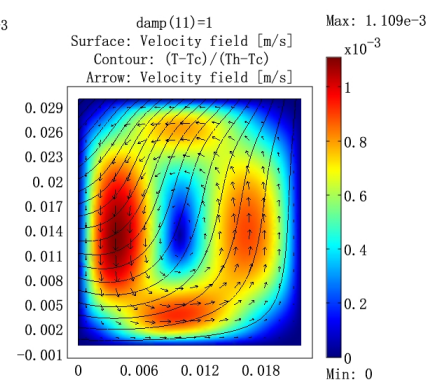

(c)

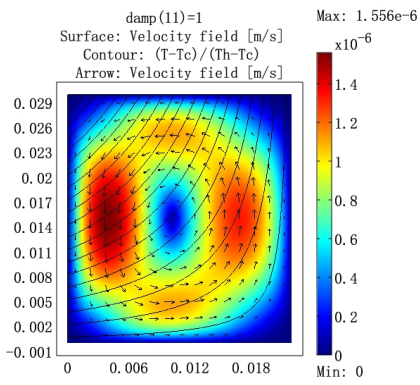

(d)
(a) $\mathrm{T}_{\mathrm{H}}=600^{\circ} \mathrm{C}, \mathrm{Tc}=500^{\circ} \mathrm{C}$
(b) $\mathrm{T}_{\mathrm{H}}=600^{\circ} \mathrm{C}, \mathrm{Tc}=550^{\circ} \mathrm{C}$
(c) $\mathrm{T}_{\mathrm{H}}=600^{\circ} \mathrm{C}, \mathrm{Tc}=575^{\circ} \mathrm{C}$
(d) $\mathrm{T}_{\mathrm{H}}=600^{\circ} \mathrm{C}$, $\mathrm{Tc}=600^{\circ} \mathrm{C}$

Fig.4 Velocity field (surface color) and velocity arrows in a porous perform subjected to temperature gradients.
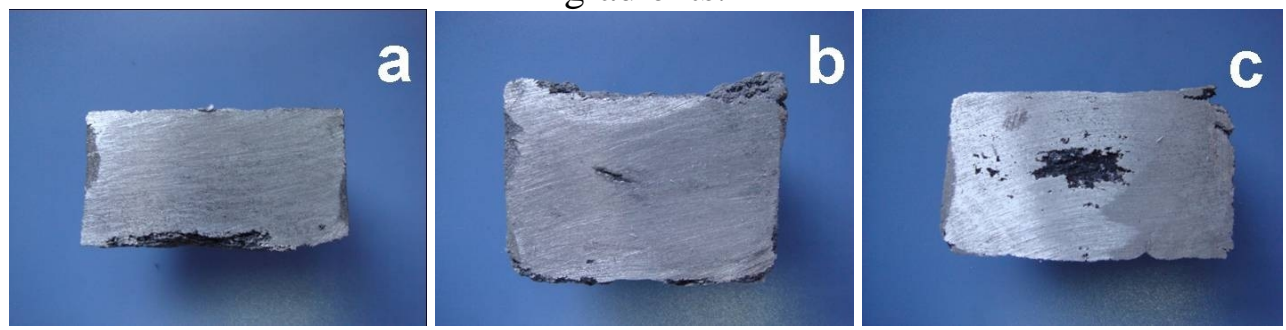

(a) $600^{\circ} \mathrm{C}$ (b) $580^{\circ} \mathrm{C}$ (c) $560^{\circ} \mathrm{C}$

Fig.5 The infiltration results of magnesium alloy into carbon fiber preform at different infiltration temperature (a) $600^{\circ} \mathrm{C}$ (b) $580^{\circ} \mathrm{C}$ (c) $560^{\circ} \mathrm{C}$

Figure 5 shows the macroscopic infiltration quality of magnesium alloy in carbon fiber preform at different preheating temperatures. It can be seen that the infiltration quality is more uniform at 600 ${ }^{\circ} \mathrm{C}$. With the decrease of temperature, the infiltration quality of the preform is worse, especially in heart. The experimental results of infiltration have a good agreement with their simulation ones.

From the above analysis, it can be found that the temperature gradients have a significant effect on the velocity distribution in the calculated region. Especially the temperature has a large effect in the near-wall region. A ring-shaped flow distribution is formed in the core, and a no-flow region appears, which is very different from the case where no temperature gradient is applied. The temperature gradient can influence the movement of infiltration by changing the dynamic viscosity and thermal conductivity of magnesium alloy melt. And the greater the temperature gradient, the impact on the seepage field is greater. Therefore, the coupling effect of temperature field and infiltration field should be considered when controlling the infiltration process. The thermodynamic properties of the local region can be adjusted by changing the temperature distribution in the prefabricated body, so as to improve the infiltration quality of the magnesium alloy in the preform.

\section{Conclusion}

(1) The mathematical model of flow and heat transfer coupling process of liquid magnesium alloy in porous preform was established, and the numerical solution of the model equation was carried out. The flow velocity field of liquid magnesium alloy under different temperature gradients was studied. The results show that the temperature gradient in the preform can change the flow property of the near wall region, and make the fluid velocity in the near wall of the preform tend to average along the flow direction.

(2) The coupled model equations of flow and heat transfer can be used to consider both the flow characteristics and the characteristics of the "adjustable" nature of the magnesium alloy liquid in the region of the coupled flow and heat transfer. 
(3) By the simulation results, temperature gradient for non-isothermal infiltrations can be optimized, which can be used to control the infiltration quality of liquid magnesium alloy in preform.

\section{References}

[1] K.K. Denga, X.J. Wang, Y.W. Wu, X.S. Hu, K. Wu and W.M. Gan: Mater. Sci. Eng. A. Vol. 543 (2012), p. 159

[2] B.N. Sahoo and S.K. Panigrahi: Mater. \& Des. Vol. 109 (2016), p. 300

[3] L.Z. Su, L.H. Qi, J.M. Zhou and H.J. Li: J. Alloy. Compd. Vol. 509 (2011), p. 775

[4] L.H. Qi, L.Z. Su, J.M. Zhou, J.T. Guana, X.H. Hou and H.J. Li: J. Alloy. Compd. Vol. 527 (2012), p. 10

[5] H.K. Ballmes, A. Klassen, C.A. Rottmair and R.F. Singer: Adv. Eng. Mater. Vol. 12 (2010), p. 577

[6] L.H. Qi, L.Z. Su, C.X. Jiang, J.M. Zhou and H.J. Li: Mater. Sci. Eng A Vol. 454-455 (2007), p. 608 